\title{
Recurrent Necrotizing Soft Tissue Infections with Atypical Pathogens after Injection Drug Abuse: Another Manifestation of the Opioid Crisis
}

\author{
Mayur Narayan, ${ }^{1}$ Jackly Juprasert, ${ }^{1}$ Caitlin Finn, ${ }^{1}$ and Philip S. Barie ${ }^{1,2}$
}

\begin{abstract}
Background: The United States is in the midst of an opioid crisis. Injection drug use is a major risk factor for necrotizing soft tissue infections (NSTI) by introducing bacteria into the soft tissues. Repetitive infection and atypical pathogens may be tangible manifestations of the opioid crisis.

Methods: We describe recidivism in two cases of NSTI involving opioid abuse and atypical pathogens at a single institution and review pertinent international literature.

Results: Repetitive NSTI is rare. Patients with chronic injection drug use, however, may be at particular risk of repetitive NSTI and infections with atypical organisms.

Conclusions: Care providers must have a heightened awareness of infections with atypical pathogens and risk of repetitive infection in patients with injection drug use. Aggressive surgical management and antibiotic coverage tailored to culture and susceptibility data are necessary for a successful short-term outcome. A successful long-term outcome mandates identification and management of co-existent behavioral health issues.
\end{abstract}

Keywords: gas gangrene; necrotizing soft tissue infection; opioid crisis

T He United States is facing an epidemic of opioid abuse, often by injection. Overdoses of these drugs, including prescription drugs, have killed more than 200,000 persons since 2000 [1]. Deaths from opioid overdose increased by $30 \%$ between 2010 and 2015 [1]. More than 64,000 Americans died from drug overdoses in 2016 alonean increase of more than $20 \%$ over the previous year $[2,3]$.

A necrotizing soft tissue infection (NSTI) is a rare, highly lethal complication of injection drug use. Rapidly fatal without treatment, NSTI carries a mortality rate of $15 \%-25 \%$ even with optimal management [4-6]. By breaching cutaneous host defenses and creating a portal of entry, injection drug use may introduce bacteria into soft tissues, increasing the risk for NSTI [7]. Further, many heroin users will resort to "skin popping" of potentially infected injection sites leading to vascular sclerosis and a microenvironment of warmth and hypoxia that allows NSTI to progress unabated [3].

The most common causative agents include (Group A) Streptococcus pyogenes (GAS), Klebsiella spp., Clostridium spp., Escherichia coli, Staphylococcus aureus, and Aero- monas hydrophila [8]. In addition, low rates of healthcare utilization among injection drug users increase the prevalence of untreated comorbid conditions that predispose to NSTI, including diabetes mellitus, obesity, immunosuppression, and non-healing wounds [8-10]. NSTI in the setting of injection drug abuse raises the potential for a complex immediate and long-term treatment course with risk of recidivism of both conditions [11]. We describe two cases of NSTI as a result of injection drug abuse: The first, another NSTI in the same location within a few weeks of apparently successful treatment of the first episode, and the second due to atypical organisms with an additional manifestation (suppurative phlebitis) during the same hospitalization.

\section{Case Reports}

Case 1

A 42-year-old female with a history of alcohol and heroin abuse was transferred from an outside hospital with two days of left upper extremity (LUE) pain, fever, and chills, which

\footnotetext{
${ }^{1}$ Department of Surgery, Division of Trauma, Burns, Acute and Critical Care, ${ }^{2}$ Department of Medicine, Division of Medical Ethics, Weill Cornell Medicine, New York, New York, USA.

Presented at the 38th Annual Meeting of the Surgical Infection Society, Westlake Village, California, April 22-25, 2018.
} 
began after injecting the site with heroin. Of note, the patient had a history of LUE NSTI with an unknown organism two months previously, which required surgical debridement at an outside hospital. The patient reported continuing to inject heroin into the LUE after treatment for the first NSTI. On presentation to us, vital signs included temperature (T) $37.0^{\circ} \mathrm{C}$, blood pressure (BP) $151 / 88 \mathrm{~mm} \mathrm{Hg}$, heart rate (HR) 104 beats/ min, and respiratory rate (RR) 22 breaths/min. The LUE was erythematous, edematous, and exquisitely tender with crepitus. Pulses were palpable and capillary refill was normal.

Laboratory studies revealed a white blood cell (WBC) count of $35,700 / \mathrm{mm}^{3}$ with $90 \%$ neutrophils and a C-reactive protein (CRP) concentration of $23.5 \mathrm{mg} / \mathrm{dL}$. Computed tomography (CT) scan showed diffuse subcutaneous edema of the distal LUE with extensive triceps intramuscular edema and gas (Figs. 1A,1B). The Laboratory Risk Indicator for Necrotizing Fasciitis (LRINEC) score [12] was nine points, indicating high suspicion for NSTI.

Microbiology data were obtainable from the first treating hospital. Vancomycin, piperacillin-tazobactam, and clindamycin were administered before emergency debridement and fasciectomy for necrosis of the triceps fascia and superficially of the muscle and copious, malodorous pus. On postoperative day (POD) 3 (Fig. 2A), a negative-pressure surgical site dressing was applied. Another debridement on POD 6 showed healthy muscle with no purulent fluid (Fig. 2B) Broadspectrum antibiotic coverage was de-escalated to cefazolin and
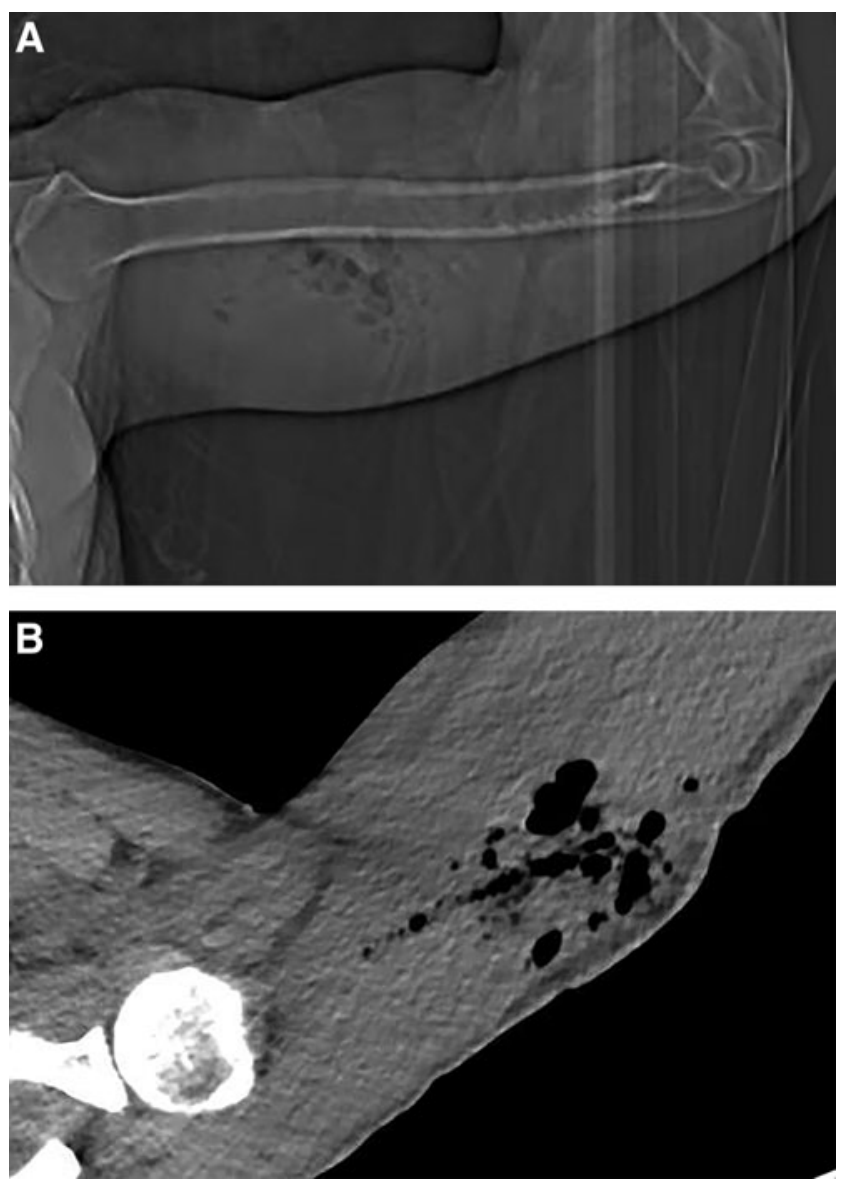

FIG. 1. Radiograph (A) and computed tomography scan (B) of left arm showing gas in soft tissues.
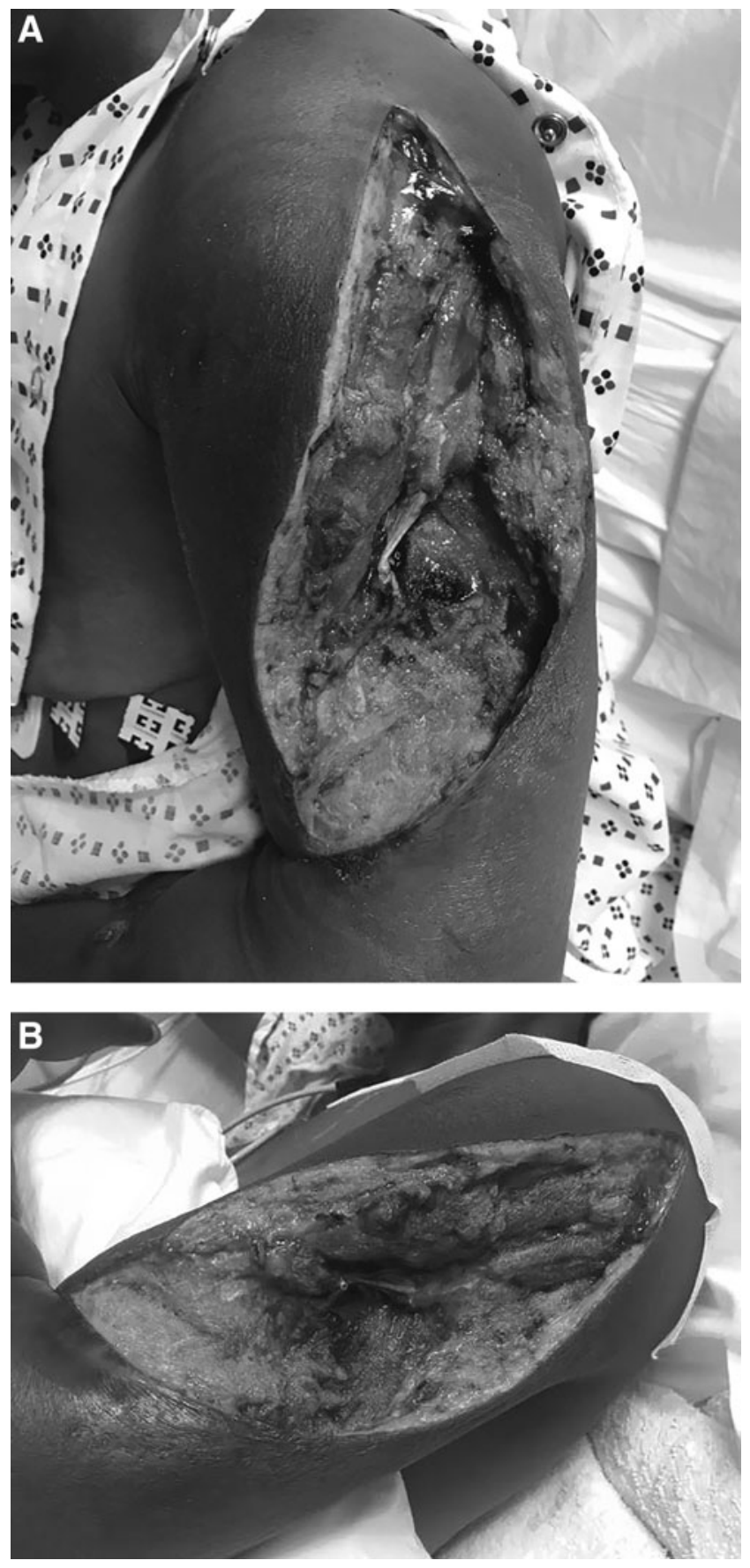

FIG. 2. Findings after initial (A) and second (B) debridement of left upper extremity in Case 1.

metronidazole after cultures yielded $E$. coli and S. anginosus. The patient never manifested signs of alcohol withdrawal.

During hospitalization, "Black Friday" packets (Fig. 3), a combination of heroin laced with fentanyl, were confiscated from the patient. Thereafter, on POD 8, the patient "eloped" from the hospital after cutting the tubing of her wound vacuum device, and was lost to follow-up.

\section{Case 2}

A 22-year old female with a history of heroin abuse was transferred from an outside hospital with LUE swelling and 


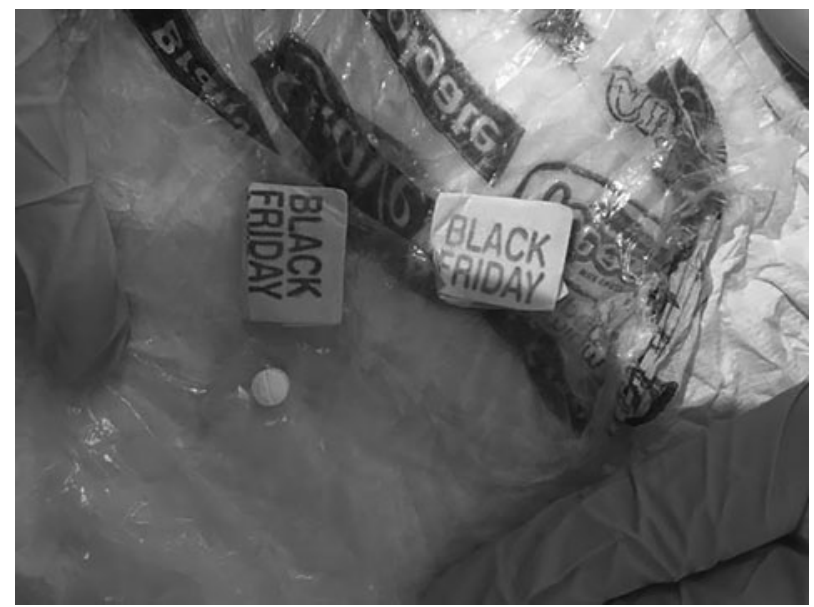

FIG. 3. "Black Friday" packets containing heroin laced with fentanyl.

pain, which began after injecting heroin five days previously. On presentation, vital signs were $\mathrm{T} 38.6^{\circ} \mathrm{C}$, BP $107 / 66 \mathrm{~mm}$ $\mathrm{Hg}$, HR 92 beats/min, and RR 14 breaths/min. On examination, there were visible "track marks" over the left forearm. The area from elbow to shoulder was erythematous, edematous, and exquisitely tender with crepitus. Peripheral pulses and capillary refill were normal. Laboratory studies revealed a WBC of $25,500 / \mathrm{mm}^{3}$ with $92.6 \%$ neutrophils. The CT scam showed diffuse subcutaneous edema of the LUE associated with subcutaneous emphysema (Fig. 4A,4B). Her LRINEC Score [12] was six points, also indicating high suspicion for NSTI.

The patient was started on the same empiric antibiotic regimen before undergoing emergency debridement and fasciectomy, which revealed necrotic muscle and fascia with copious, malodorous pus. She was returned to the operating room the following day for washout and debridement of multiple abscesses of the upper arm to the rotator cuff and subacromial space. Intra-operative cultures from the index operation showed S. anginosus, Prevotella buccae, and Bilophila wadsworthia, all part of normal oral flora and all susceptible to penicillin G. Leukocytosis resolved but recurred on hospital day (HD) 9; penicillin was changed to meropenem, but re-operation revealed only healthy granulation tissue, allowing discontinuation of the antibiotic agent. A tissue expander was placed, enabling primary surgical site closure five days later.

On HD 20, the temperature of the patient was $38.6^{\circ} \mathrm{C}$, and she was placed on empiric meropenem with blood cultures subsequently positive for Burkholderia cepacia complex. Despite therapy, the patient continued to be febrile ( $\mathrm{T}$ max $39.4^{\circ} \mathrm{C}$ ). Right upper extremity (RUE) ultrasonography revealed superficial thrombophlebitis. On HD 25, excision of a thrombosed RUE cephalic vein was consistent with suppurative thrombophlebitis (Fig. 5). Parenteral vancomycin was added.

On HD 29, recrudescent fever $\left(\mathrm{T} 39.3^{\circ} \mathrm{C}\right)$ developed with new blood cultures positive for both Candida tropicalis

FIG. 4. Coronal (A) and axial (B) computed tomography of left upper extremity showing subcutaneous edema associated with emphysema in Case 2.
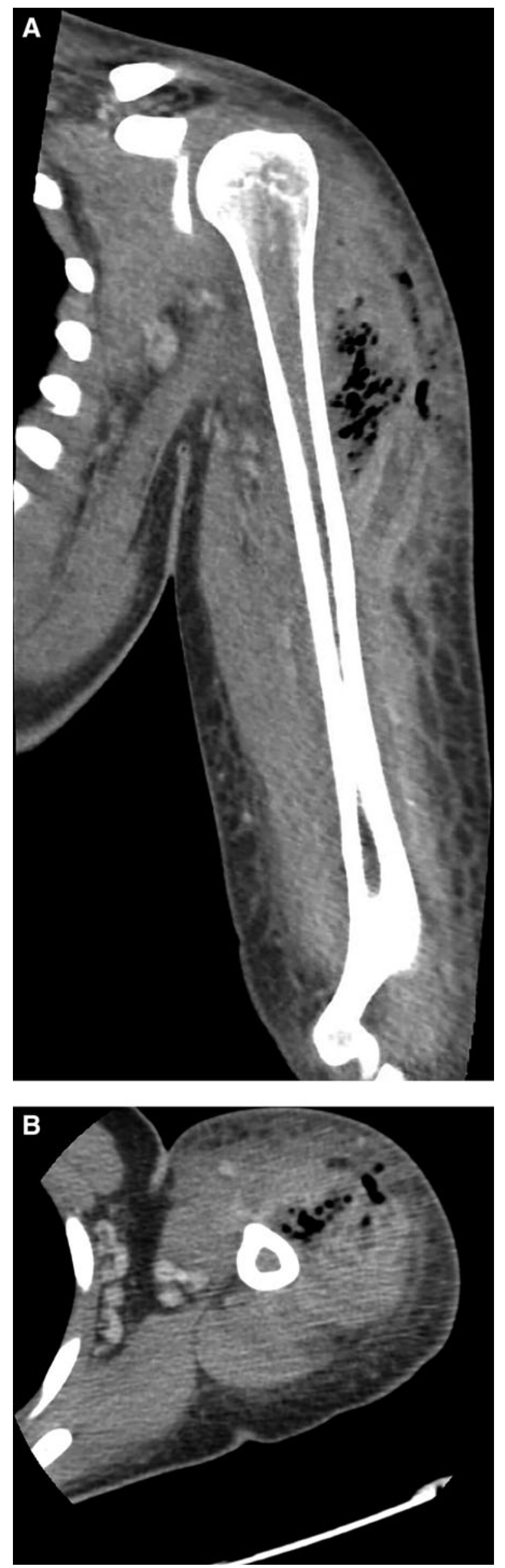


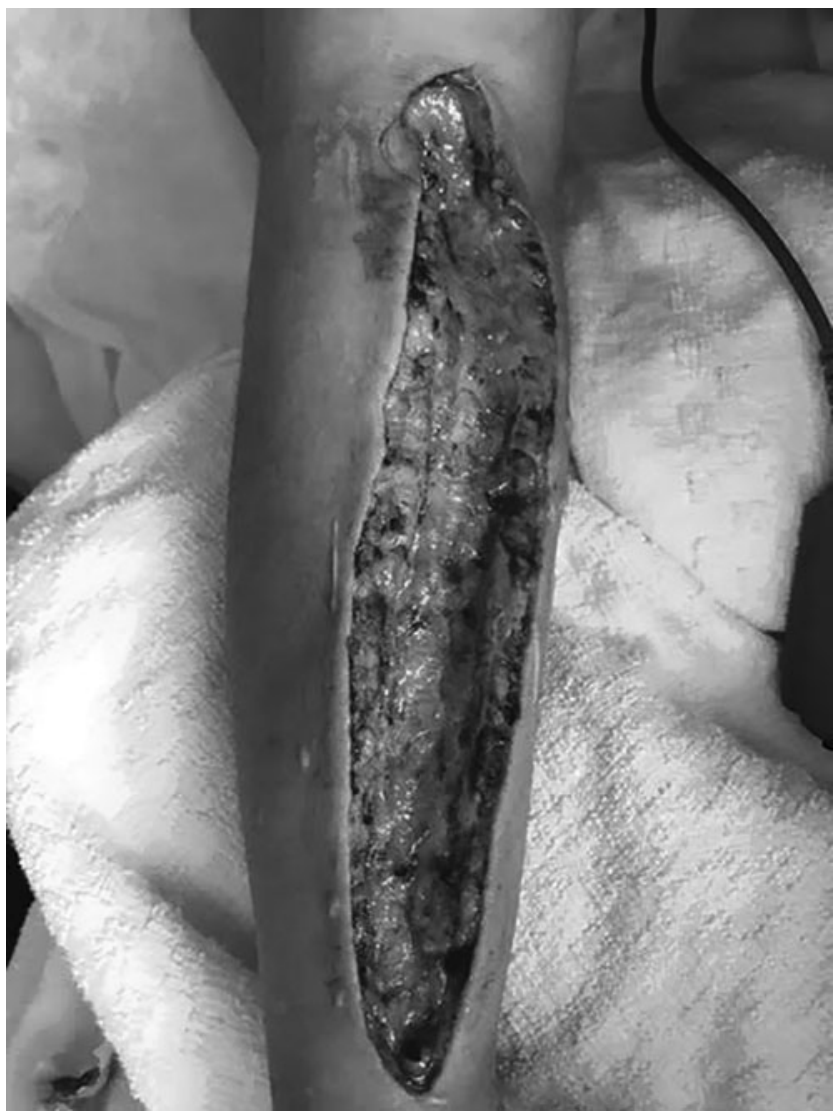

FIG. 5. Debrided right forearm with excision of cephalic vein in Case 2.

and $C$. albicans, for which treatment was with fluconazole. Thereafter the patient had multiple positive blood cultures, which variably grew a multiplicity of C. tropicalis, C. albicans, Stenotrophomonas maltophilia, and Abiotrophia defectiva. Extensive evaluation, including transthoracic echocardiogram, CT of the abdomen/pelvis, and ophthalmic funduscopy did not identify a source. Subsequently, when the patient was discovered to be injecting her own feces intravenously, antimicrobial and psychiatric intervention was undertaken. Thereafter, under continuous supervision, blood cultures remained negative and appropriate antimicrobial therapy controlled the blood stream infections.

\section{Discussion}

To our knowledge, this is the first case series of repeat NSTI in injection drug users. These cases are noteworthy considering the increase of opioid abuse in the United States. Drug abuse was commonly believed to be an inner-city problem, but is now affecting other socioeconomic classes in increasing numbers. Expanding use of heroin and other injectable drugs has been related to the increasing abuse of prescription drugs such as oxycodone (OxyContin, Purdue Pharma, L.P., Stamford, CT), hydrocodone/acetaminophen, and fentanyl.

Opioid addition is considered a chronic, relapsingremitting disorder $[13,14]$. Opioid use is notoriously difficult to treat, and the path to recovery is often marred by relapses. Smyth et al. [15] reported that $91 \%$ of patients with opioid addition relapsed after inpatient drug rehabilitation; 59\% re- lapsed within one week. Even after a non-fatal overdose, $91 \%$ of patients subsequently receive prescription opioids, and $17 \%$ will experience a second overdose within two years [16].

We highlight the importance of injection drug use as a major risk factor for NSTI and the potential for recidivism. In Case 1, the patient reported continued injection drug use in the affected arm between episodes of NSTI. In Case 2, the patient had discrete, consecutive episodes of NSTI in both upper extremities, both caused by atypical pathogens, for which injection drug use is known to predispose. Injection drug use was associated with more than $40 \%$ of cases of NSTI because of community-acquired methicillin-resistant $S$. $a u$ reus (MRSA) in one community sample [17].

In Case 1, intra-operative cultures demonstrated infection with $S$. anginosus, a rare organism in NSTI although it demonstrates propensity to form abscesses in soft tissue. $S$. anginosus has been implicated in antecubital abscesses in injection drug users [18] and craniofacial NSTI [19-21]. In Case 2, the patient's intra-operative cultures showed polymicrobial normal oral flora [22], although such organisms are known to cause soft tissue infections [23]. Thereafter. the patient was found to have B. cepacia complex bacteremia, leading to the diagnosis of suppurative thrombophlebitis, itself a rarity, because $B$. cepacia has been reported only once previously [24]. Throughout hospitalization, the Case 2 patient had multiple nosocomial blood stream infections with several species of yeast and bacteria despite ostensibly appropriate antibiotic coverage.

Reports of repeated episodes of NSTI in a single patient are few. Wong et al. [25] described recurrent NSTI caused by MRSA in a 46-year-old female with poorly controlled diabetes mellitus and chronic glucocorticoid use. Kuzdan et al. [26] described a fatal case of recurrent NSTI due to $S$. pyogenes in a child with hereditary sensory and autonomic neuropathy type IV. Kumar [27] described a case of recurrent NSTI in a patient with complement C4 deficiency. Mackay et al. described recurrent inflammation in a site of previous NSTI in a patient during chemotherapy [28]. Adelson et al. [29] reported a case of NSTI of the vulva in a patient with obesity and diabetes mellitus. Each of these patients had at least one major risk factor for NSTI. To our knowledge, this is the first report of recurrent NSTI in patients who injected illicit opioids.

In the assessment of patients who use injection drugs, it is important to have a high index of suspicion for NSTI, given their increased risk. In addition, an episode of NSTI may carry a risk of recurrent infection because of the likely repeat exposure to bacteria through continuing injection drug use. The management of NSTI in patients who use opioids should involve particular attention to identification and management of coexistent behavioral health problems, multimodality (opioid-sparing) post-operative pain management, and safe discharge planning [30], including inpatient rehabilitation of appropriate patients.

\section{Author Disclosure Statement}

No competing financial interests exist.

\section{References}

1. Rudd RA, Seth P, David F, Scholl L. Increases in drug and opioid-involved overdose deaths - United States, 2010-2015. MMWR Morb Mortal Wkly Rep 2016;65:1445-1452. 
2. Hedegaard H, Miniño AM, Warner M. Drug overdose deaths in the United States, 1999-2017. NCHS Data Brief 2018;329:1-8.

3. Bauman ZM, Morizio K, Singer M, et al. The heroin epidemic in America: A surgeon's perspective. 2019;20:351-358.

4. Khamnuan P, Chongruksut W, Jearwattanakanok K, et al. Necrotizing fasciitis: Risk factors of mortality. Risk Manag Healthc Policy 2015;8:1-7.

5. Rajput A, Waseem, Samad A, et al. Mortality in necrotizing fasciitis. J Ayub Med Coll Abbottabad 2008;20:96-98.

6. Huang KF, Hung MH, Lin YS, et al. Independent predictors of mortality for necrotizing fasciitis: A retrospective analysis in a single institution. J Trauma 2011; 71:467-473.

7. Chen JL, Fullerton KE, Flynn NM. Necrotizing fasciitis associated with injection drug use. Clin Infect Dis 2001;33: 6-15.

8. Stevens DL, Bryant AE. Necrotizing soft-tissue infections. N Engl J Med 2017;377:2253-2265.

9. Maragh-Bass AC, Powell C, Park J, et al. Sociodemographic and access-related correlates of health-care utilization among African American injection drug users: The BESURE study. J Ethn Subst Abuse 2017;16:344-362.

10. Chitwood DD, Sanchez J, Comerford M, McCoy CB. Primary preventive health care among injection drug users, other sustained drug users, and non-users. Subst Use Misuse 2001;36:807-824.

11. Hakkarainen TW, Kopari NM, Pham TN, Evans HL. Necrotizing soft tissue infections: Review and current concepts in treatment, systems of care, and outcomes. Curr Probl Surg 2014;51:344-362.

12. Wong $\mathrm{CH}$, Khin LW, Heng KS, lt al. The LRINEC (laboratory Risk Indicator for Necrotizing Fasciitis) score: A tool for distinguishing necrotizing fasciitis from other soft tissue infections. Crit Care Med 2004;32:1535-1541.

13. Hser YI, Evans E, Grella C, et al. Long-term course of opioid addiction. Harvard Rev Psychiatr 2015;23:76-89.

14. Volkow ND, Collins FS. The role of science in addressing the opioid crisis. N Engl J Med 2017;377:391-394.

15. Smyth BP, Barry J, Keenan E, Ducray K. Lapse and relapse following inpatient treatment of opiate dependence. Irish Med J 2010;103:176-179.

16. Larochelle MR, Liebschutz JM, Zhang F, et al. Opioid prescribing after nonfatal overdose and association with repeated overdose: A cohort study. Ann Intern Med 2016; 164:1-9.

17. Miller LG, Perdreau-Remington F, Rieg G, et al. Necrotizing fasciitis caused by community-associated methicillinresistant Staphylococcus aureus in Los Angeles. N Engl J Med 2005;352:1445-1453.
18. Stocker E, Cortes E, Pema K, et al. Streptococcus milleri as a cause of antecubital abscess and bacteremia in intravenous drug abusers. South Med J 1994;87:95-96.

19. Park E, Hirsch EM, Steinberg JP, Olsson AB. Ascending necrotizing fasciitis of the face following odontogenic infection. J Craniofac Surg 2012;23:e211-e214.

20. Gausepohl JS, Wagner JG. Survival from cervical necrotizing fasciitis. West J Emerg Med 2015;16:172-174.

21. Shield DR, Servat J, Paul S, et al. Periocular necrotizing fasciitis causing blindness. JAMA Ophthalmol 2013;131: 1225-1227.

22. Baron EJ. Bilophila wadsworthia: A unique gram-negative anaerobic rod. Anaerobe 1997;3:83-86.

23. Finegold S, Summanen P, Hunt Gerardo S, Baron E. Clinical importance of Bilophila wadsworthia. Eur J Clin Microbiol Infect Dis 1992;11:1058-1063.

24. Marioni G, Rinaldi R, Ottaviano G, et al. Cervical necrotizing fasciitis: novel clinical presentation of Burkholderia cepacia infection. J Infect 2006;20;53:e219-e222.

25. Wong CH, Tan SH, Kurup A, Tan AB. Recurrent necrotizing fasciitis caused by methicillin-resistant Staphylococcus aureus. Eur J Clin Microbiol Infect Dis 2004;23:909-911.

26. Kuzdan C, Soysal A, Altinkanat G, et al. Recurrent fatal necrotizing fasciitis due to Streptococcus pyogenes in a child with hereditary sensory and automic neuropathy type IV. Jpn J Infect Dis 2011;64:147-149.

27. Kumar PD. Recurrent necrotizing fasciitis in complement C4 deficiency. Ann Intern Med 2001;135:635.

28. Mackay HJ, Williamson EC, Vasey P, Harnett AN. Recurrent inflammation in a site of previous necrotising fasciitis during intravenous CMF chemotherapy. Ann Oncol 1999;10:1101-1103.

29. Adelson MD, Joret DM, Gordon LP, Osborne NG. Recurrent necrotizing fasciitis of the vulva. A case report. J Reprod Med 1991;36:818-822.

30. Wagner KD, Liu L, Davidson PJ, et al. Association between non-fatal opioid overdose and encounters with healthcare and criminal justice systems: Identifying opportunities for intervention. Drug Alcohol Depend 2015;153:215-220.

Address correspondence to: Dr. Philip S. Barie Department of Surgery, P713A New York-Presbyterian Hospital Weill Cornell Medical Center 525 East 68th Street New York, NY 10065 USA

E-mail: pbarie@med.cornell.edu 